\title{
Divertículo único de yeyuno con obstrucción intestinal y abdomen agudo
}

\author{
Unique diverticulum of jejunum with intestinal obstruction \\ and acute abdomen
}

\author{
Ricardo A. Villarreal ${ }^{1}$, Eric E. Vinck ${ }^{2}$, Luis Felipe Cabrera ${ }^{2}$, Andrés C. Zuchini $^{2}$, Cristina Jiménez ${ }^{3}$ \\ 1 Departamento de Cirugía Gastrointestinal y Endoscópico, Universidad El Bosque, Bogotá, D.C., Colombia \\ 2 Departamento de Cirugía General, Universidad El Bosque, Bogotá, D.C., Colombia \\ 3 Médica interna, Departamento de Cirugía General, Universidad El Bosque, Bogotá, D.C., Colombia
}

\section{Resumen}

Introducción. El hallazgo de divertículos en el intestino delgado es poco usual, y se presentan principalmente en el yeyuno y en el íleon. Tienen una incidencia relativamente baja, aproximadamente, de o,06 a $5 \%$ en autopsias y de 0,5 a 2,3 \% como hallazgos radiológicos. Predominan en la sexta y la séptima década de la vida y en el sexo masculino.

Presentación de caso. Se trata de una paciente de sexo femenino de 90 años, que ingresó al servicio de urgencias por un cuadro clínico de un día de dolor en el hemiabdomen derecho irradiado al hipogastrio y asociado con múltiples episodios de emesis. Se hospitalizó para estudios paraclínicos y, durante la observación, presentó deterioro clínico, por lo que fue sometida a una laparotomía exploratoria, y se encontró un divertículo único de yeyuno. Discusión. Los divertículos de intestino delgado son poco frecuentes, y comprometen solo la mucosa y submucosa. Del total de casos, del o,9 a I \% corresponden a divertículos del yeyuno. Estos pueden asociarse con divertículos en otra parte del tubo digestivo, como esófago (2,3\%), duodeno (30 \%) o colon (6I \%). El manejo de esta enfermedad depende de cada paciente.

Palabras clave: yeyuno; divertículo; obstrucción intestinal; abdomen agudo; laparotomía.

\begin{abstract}
Small intestine diverticulum is a rare finding; it presents more often in the jejunum and ileum. The incidence in autopsies is low (0.06-5\%), and 0.5-2.3\% as a radiology finding. It is most often found in the 7 th decade and in men. A 90-year-old female patient presented with a clinical picture of one day consisting of acute right abdominal pain that radiates to the hypogastrium accompanied by multiple episodes of vomiting. The patient is admitted and a battery of tests are ordered; during observation the patient quickly deteriorates and is taken to an emergency laparotomy, finding a single jejunal diverticulum. Small intestine diverticuli are rare surgical findings (o.9-I\%) that involve only the mucosa and submucosa. The majority of small intestinal diverticuli are found in the jejunum.

Fecha de recibido: 9/03/2018. Fecha aceptación: 1/06/2018.

Correspondencia: Eric Vinck, MD, Bogotá, D.C., Colombia

Correo electrónico: evinck518@gmail.com

Citar como: Villarreal RA, Vinck EE, Cabrera LF, Zuchini AC, Jiménez C. Divertículo único de yeyuno con obstrucción intestinal y abdomen agudo. Rev Colomb Cir. 2019;34:69-74. https://doi.org/10.30944/20117582.100

Este es un artículo de acceso abierto bajo una Licencia Creative Commons - BY-NC-ND https://creativecommons.org/licenses/by-nc-nd/4.0/deed.es
\end{abstract}


These can be associated with diverticuli in other areas of the GI tract: stomach (2.3\%), duodenum (30\%) and colon (6I\%). The management approach depends on each patient's clinical presentation.

Key words: jejunum; diverticulum; intestinal obstruction; abdomen, acute; laparotomy.

\section{Introducción}

El hallazgo de divertículos en el intestino delgado es poco usual, y su presentación es más frecuente en el yeyuno y en el íleon. En términos generales, estos divertículos presentan una incidencia, aproximadamente, de 0,06 a $5 \%$ en autopsias y de 0,5 a 2,3 \% como hallazgos radiológicos. Son más predominantes en la sexta y en la séptima década de la vida, y según algunos autores, predomina en el sexo masculino ${ }^{\mathrm{I}-9}$.

\section{Etiología}

Estos divertículos pueden ser congénitos o adquiridos. El único congénito es el divertículo de Meckel, que se localiza en el borde antimesentérico del intestino delgado, aproximadamente, de 40 a $80 \mathrm{~cm}$ de la válvula ileocecal. El resto de divertículos en esta localización, se caracterizan por ser pseudodivertículos, y se localizan en el borde mesentérico.

\section{Historia}

Los divertículos del intestino delgado fueron descritos en I794 por Sommervit. Posteriormente, en 1807 , Cooper describió por primera vez los divertículos en yeyuno. Quienes reportaron por primera vez las complicaciones de esta enfermedad fueron Virchow en I853 y Osler en I88I. En 1906, Gordinier y Sampson describieron el primer paciente que fue intervenido quirúrgicamente por presentar diverticulosis del yeyuno, ya que previamente este tipo de enfermedades sólo se identificaban en las autopsias. En 1920 se reportaron los primeros cinco casos de diverticulosis del intestino delgado identificados mediante radiología ${ }^{9-13}$.

\section{Epidemiología}

Los divertículos de intestino delgado son poco frecuentes, con una incidencia de 0,06 a $5 \%$, y comprometen solo la mucosa y submucosa. Del total de casos, 0,9 a I \% corresponden a divertículos del yeyuno ${ }^{1,2,4,4,13}$. Estos pueden asociarse a divertículos en otra parte del tubo digestivo, como esófago $(2,3 \%)$, duodeno (30 \%) y colon $(6 \mathrm{I} \%)^{\mathrm{I}, 2, \mathrm{II}-\mathrm{I} 3)}$. Su incidencia disminuye progresivamente en regiones distales al ángulo de Treitz y la prevalencia aumenta con la edad, sobre todo en pacientes en la sexta y la séptima décadas de la vida. Existen varios estudios que describen mayor incidencia en hombres que en mujeres, aunque esto no es muy claro. En cuanto a los divertículos de intestino delgado, son más frecuentes en yeyuno (80 \%), seguido de íleon (I5 $\%)$ y en ambas localizaciones (5\% ${ }^{2,8-13}$.

\section{Fisiopatología}

Los divertículos pueden ser congénitos o adquiridos. El resto de divertículos en esta localización se caracterizan por ser pseudodivertículos y se localizan en el borde mesentérico ${ }^{6, \mathrm{II}-13}$. Estos divertículos se forman por la herniación de la capa mucosa y submucosa en los puntos de menor resistencia a la presión dentro de la luz intestinal, como la entrada de los vasos sanguíneos; por eso, su principal complicación es la hemorragia. La formación de estos divertículos permite la acumulación de residuos, la proliferación bacteriana y la formación de 'enterolitos', lo cual aumenta el riesgo de diverticulitis y perforación. Los divertículos del intestino delgado tienden a ser múltiples; en la región proximal, suelen presentar mayor tamaño y cantidad que en la región caudal $15,9, \mathrm{II}$.

En cuanto a la fisiopatología, no está muy claro el mecanismo por el que se producen. Sin embargo, la teoría más aceptada es la que describe una debilidad del plexo mientérico que causa contracciones irregulares del intestino, lo cual genera un aumento en la presión dentro de la luz intestinal, lo que favorece la formación de di- 
vertículos en los puntos más débiles del intestino 1,2,5,7,7,13. Esta enfermedad se relaciona con esclerosis sistémica, miopatías y neuropatía visceral. La incidencia de estos divertículos en pacientes con esclerosis múltiple es de $42 \%$, así como en casos de síndrome de Elhers-Danlos o miastenia gravis. Esta última se relaciona, posiblemente, con un aumento de la presión dentro de la luz intestinal o con el uso de medicamentos anticolinesterasa ${ }^{1,6,7,9,11}$.

\section{Presentación clínica}

Por lo general, los divertículos del yeyuno son asintomáticos (60\%). Sin embargo, una vez se tornan sintomáticos, su tratamiento es quirúrgico en el IOo \% de los casos; el 93,3\% de estos requieren resección del segmento intestinal donde se halla el divertículo. Cuando se presentan, en 30 $\%$ de los casos, los síntomas pueden ser inespecíficos, como dolor abdominal intermitente (principalmente en el cuadrante superior izquierdo), cambios en las deposiciones, esteatorrea y síntomas de malabsorción por movimientos peristálticos asincrónicos, dilatación de divertículos, estasis de contenido intestinal o proliferación bacteriana. Aproximadamente, el Io \% de los pacientes llega a desarrollar síntomas asociados con alguna complicación, como anemia por sangrado o por deficiencia de vitamina $\mathrm{B}_{\mathrm{I} 2}$ secundaria a la proliferación bacteriana $a^{2,7,8,9,11,12}$.

Las principales complicaciones son obstrucción (6\%), hemorragia, diverticulitis, perforación y vólvulos. Otras menos frecuentes, son absceso abdominal, y neumoperitoneo crónico y repetitivo por microperforación. Las complicaciones aumentan la tasa de mortalidad hasta en $24 \%$, teniendo en cuenta que los divertículos tienen cuatro veces más posibilidades de presentar complicaciones cuando se localizan en el yeyuno $^{3,4,10,12,13}$.

\section{Diagnóstico}

El diagnóstico de esta entidad es incidental en la mayoría de los casos. Sin embargo, se han encontrado en colangiopancreatografía retrógrada endoscópica (CPRE) o enteroscopia y, también, como hallazgo incidental en la tomografía axial (TC) abdominal.

Otro procedimiento para diagnosticar esta entidad es la endoscopia de avance, útil para evaluar la parte distal del duodeno y la proximal del yeyuno, ya que con esta se puede observar de 90 a $150 \mathrm{~cm}$ después de ligamento de Treitz.

Por otra parte, está la enteroscopia de doble balón, que puede avanzar de 240 a $360 \mathrm{~cm}$ distal al píloro y de 102 a I $40 \mathrm{~cm}$ proximal a la válvula ileocecal con abordaje rectal. La videocápsula endoscópica, que se usa cuando la endoscopia y la colonoscopia son normales, tiene un éxito de 38 a $83 \%$ para el diagnóstico de divertículos yeyunales ${ }^{1,6,13}$. No se recomienda su uso en caso de divertículos grandes, ya que existe el riesgo de que quede atrapada en uno de ellos ${ }^{\mathrm{I}}$. La enteroscopia intraoperatoria -con una mortalidad del I7 \% - se usa solo cuando los resultados de la enteroscopia de doble balón y de la videocápsula son negativos.

Cuando se presenta hemorragia activa, se acude a la arteriografía mesentérica o la gammagrafía con enterocitos marcados. Sin embargo, para que el sangrado se pueda observar, se requiere un flujo mínimo de o,5 a I $\mathrm{ml}$ por minuto en la arteriografía, y uno de o,I $\mathrm{ml}$ por minuto en la gammagrafía. En casos de difícil diagnóstico con complicaciones, se utiliza la laparotomía exploratoria como abordaje inicial ${ }^{4,5,6,13}$.

\section{Tratamiento}

Generalmente, el tratamiento es la resección quirúrgica del divertículo o del segmento involucrado, con anastomosis primaria del mismo. Los divertículos asintomáticos del yeyuno no requieren tratamiento quirúrgico, y los pacientes con sintomatología crónica pueden manejarse de forma conservadora. Solo se considera la cirugía si persisten con sintomatología. En caso de perforación, se practica una laparotomía exploratoria. En caso de diverticulitis no perforada, se sugiere tratamiento antibiótico y reposo intestinal; cuando hay abscesos intraabdominales, está indicado el drenaje percutáneo. 
Algunos autores están a favor de las resecciones profilácticas debido a la gravedad de las complicaciones. Sin embargo, no se han demostrado mayores beneficios con ellas, teniendo en cuenta que la mayoría de los pacientes son de edad avanzada y tienen múltiples comorbilidades ${ }^{1,2,3,8,12}$. Cuando se requiere la resección, no debe ser extensa para evitar el síndrome de intestino corto ${ }^{5,6}$. En pacientes con enfermedad no complicada, está indicado el tratamiento conservador, que incluye medidas como dieta hiperproteica, suplementos vitamínicos, antiespasmódicos, antidiarreicos, antiácidos y analgésicos, con tasas de éxito de hasta el $75 \%$.

\section{Presentación del caso}

Se presenta un caso de divertículo único del yeyuno, que cursó con obstrucción intestinal y abdomen agudo.

Se trata de una mujer de 90 años de edad que ingresó al servicio de urgencias por presentar un cuadro clínico de un día de evolución, consistente en dolor en el hemiabdomen derecho irradiado al hipogastrio, asociado con tres episodios de emesis de contenido bilioso.

Tenía antecedentes de hipertensión arterial sistémica, reflujo gastroesofágico, osteoporosis, artrosis e hipoacusia, y no tenía antecedentes de cirugía abdominal. Ingresó con tensión arterial de I42/64 mm $\mathrm{Hg}$, con dolor a la palpación profunda en hipogastrio, región periumbilical e hipocondrio derecho, y sin signos de irritación peritoneal.

En la ecografía abdominal se observó distensión de las asas intestinales en los flancos, sin líquido libre en la cavidad abdominal. Los exámenes de laboratorio de perfil hepático, hemograma, función renal y tiempos de coagulación, estaban dentro de límites normales.

Por persistencia del cuadro clínico, se solicitó TC abdominal con contraste, en la que se apreció un íleo parcialmente obstructivo, hernia diafragmática derecha, dilatación esofágica indicativa de acalasia y derrame pleural y atelectasias en ambas bases pulmonares (figuras I y 2).

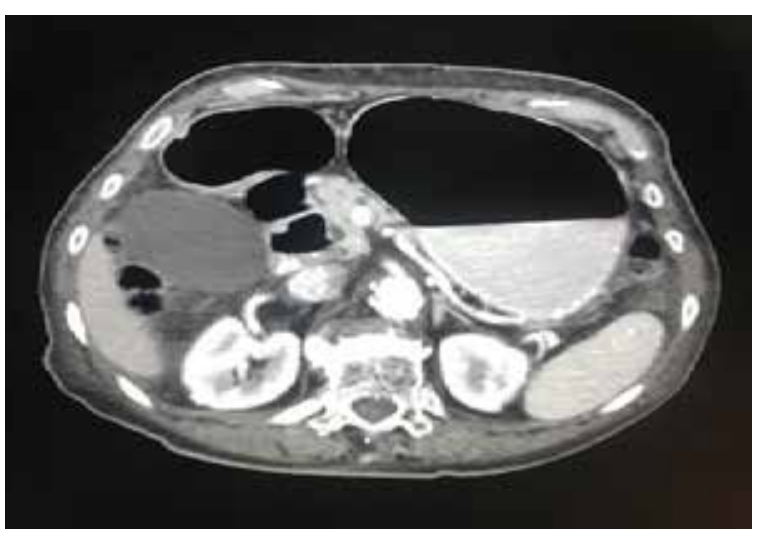

Figura 1. TC abdominal con contraste: distensión gástrica, y de la primera y la segunda porciones del duodeno

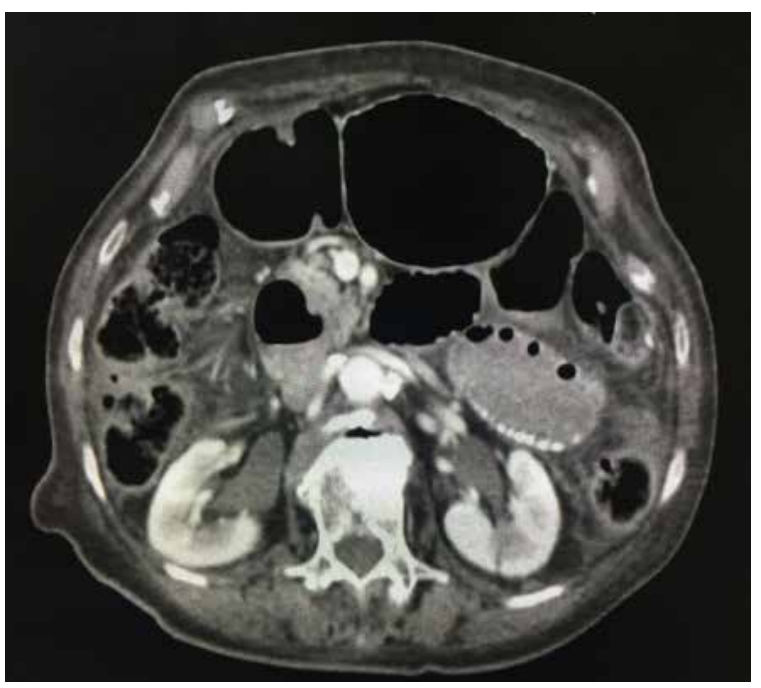

Figura 2. TC abdominal con contraste: importante distensión de asas intestinales sin divertículos evidentes a este nivel

Se hospitalizó para observación y, 48 horas después presentó signos de irritación peritoneal más distensión abdominal importante, por lo que se sometió a laparotomía. En el yeyuno proximal, se encontró una zona de transición por vólvulo del asa intestinal con giro sobre su eje longitudinal, y dilatación proximal y distal de las asas intestinales delgadas. A Io $\mathrm{cm}$ de ángulo de 
Treitz, se encontró un divertículo en yeyuno, sin serosa, comprometido por el vólvulo e hipoperfusión asociada (figura 3). Con estos hallazgos, se decidió practicar una resección intestinal con anastomosis yeyuno-yeyuno mediante sutura lineal mecánica (figuras 4 y 5).

La paciente completó el tratamiento antibiótico posoperatorio de siete días y su tolerancia a la vía oral fue adecuada.

En el estudio de histopatología se encontró un fragmento de intestino en forma de saco de 2,3 $\mathrm{x} \mathrm{I,8} \mathrm{x} \mathrm{I,2} \mathrm{cm,} \mathrm{con} \mathrm{congestión} \mathrm{vascular,} \mathrm{sin} \mathrm{capa}$ muscular propia, correspondiente a un divertículo adquirido de intestino delgado. Un mes después de la cirugía, la paciente presentaba una evolución clínica adecuada, se encontraba asintomática, recibiendo dieta normal y buen tránsito intestinal yeyuno-yeyuno.

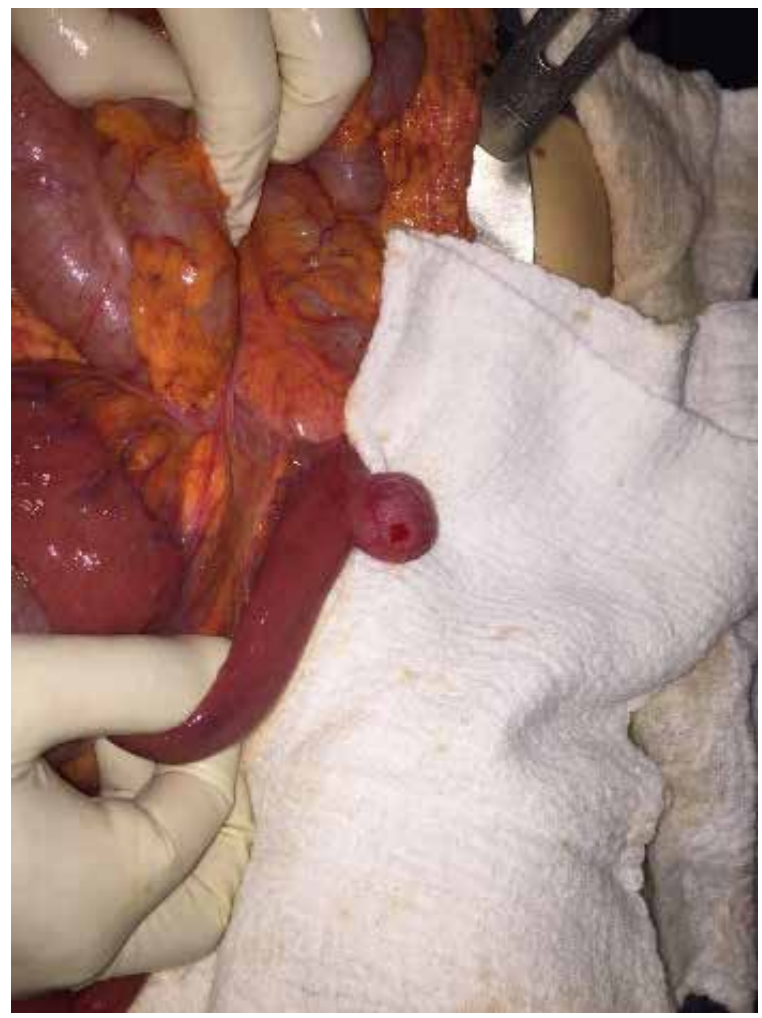

Figura 3. Divertículo único de yeyuno, de $2,5 \mathrm{~cm}$ de diámetro

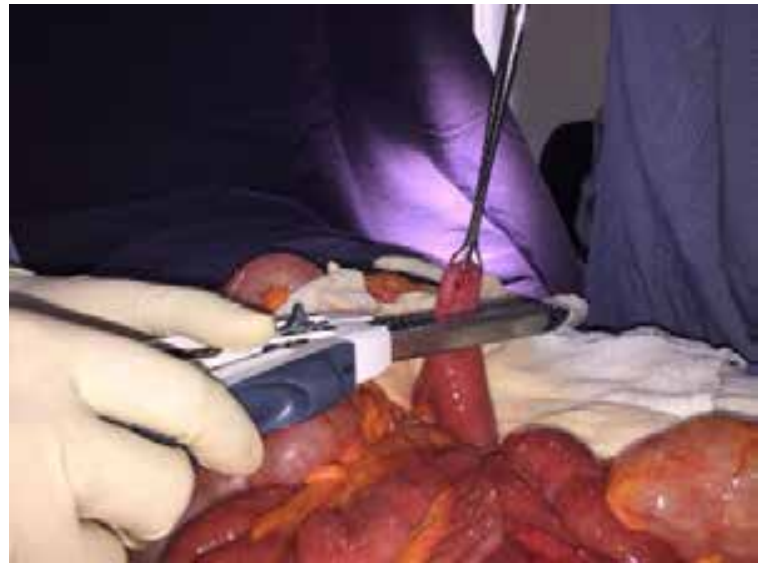

Figura 4. Sutura mecánica para resecar el divertículo

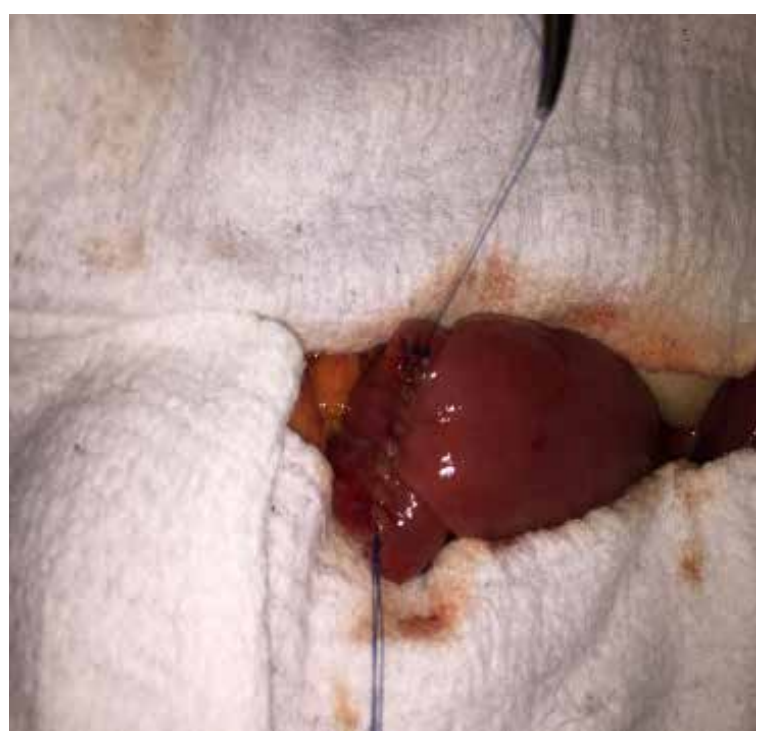

Figura 5. Sutura de refuerzo posterior a la resección y la anastomosis

\section{Conclusión}

Los divertículos del yeyuno constituyen un hallazgo poco frecuente y, a pesar de los avances en imágenes diagnósticas, la gran mayoría se descubre durante la intervención quirúrgica por abdomen agudo como complicación del divertículo. El manejo de esta enfermedad, por lo general, es la resección segmentaria de la porción intestinal afectada por el divertículo; por lo tanto, el cirujano debe incluir esta alteración en su lista 
de diagnósticos diferenciales, ya que sus complicaciones son importantes.

\section{Conflictos de interés. Ninguno declarado}

\section{Fuentes de financiación. Autofinanciado}

\section{Referencias}

I. Blake-Siemsen JC, Kortright-Farías M, Casale-Menier DR, Gámez-Araújo J. Sangrado digestivo por divertículos yeyunales: reporte de un caso y revisión de la literatura. Cir Cir. 20I7;85:34-9. doi: Io.IOI6/j.circir.20I6.II.OII

2. Romera-Barba E, Gálvez-Pastor S, Navarro-García MI, Torregrosa-Pérez MN, Sánchez-Pérez A, Vásquez-Rojas JL. Diverticulosis yeyunal: una causa rara de obstrucción intestinal. Gastroenterol Hepatol. 20I7;40:399-4OI. doi: og/Io.I6/j.gastrohep.20I6.04.0I6

3. Jochmans I, Pirenne J. Jejunal diverticulosis with midgut volvulus and intestinal malrotation. N Eng J Med. 20I6;375:e2. doi: I0.IO56/NEJMicmI5III65

4. Walter BM, Winker J, Wagner M, Jung A, Strebel H, Born P. Complicated jejunal diverticulosis -a rare but important diagnosis to consider in abdominal pain: A report of three cases. Zeitschrift für Gastroenterologie. 2016;220:562-5.

5. Kwak JY, Park EH, Park CS, Kim JH, Han MS, Kwak JH. Uncomplicated jejunal diverticulosis with pneumoperitoneum. Ann Surg Treat Res. 2016;90:346-9. doi: I0.4I74/astr.20I6.90.6.346

6. Ghrissi R, Harbi H, Elghali MA, Belhajkhlifa MH, Letaief MR. Jejunal diverticulosis: A rare case of intesti- nal obstruction. J Surg Case Rep. 20I6;20I6:rjvi76. doi: IO.I093/jscr/rjvi76

7. Hanna C, Mullinax J, Friedman MS, Sánchez J. Jejunal diverticulosis found in a patient with long-standing pneumoperitoneum and pseudo-obstruction on imaging: A case report. Gastroenterol Report. 2016;4:33740. doi: Io.Io93/gastro/govo33

8. Tenreiro N, Moreira H, Silva S, Marques R, Monteiro A, Gaspar J, et al. Jejunoileal diverticulosis, a rare cause of ileal perforation-Case report. Ann Med Surg. 2016;6:56-9. doi: I0.IoI6/j.amsu.2016.0I.089

9. Hevia M, Quera R, Soto L, Regueira T, O'Brien A, Larach A, et al. Diverticulitis aguda de intestino delgado en un paciente con enfermedad de Crohn: Report of one case. Rev Méd Chil. 20I7;I45:397-4OI. Doi: org/I0.4067/So034-988720170003000I6

Io. Bratting-Cantão CA, Ribeiro-Feitosa MR, Godinho M, Scarpelini S, Féres O, Ribeiro da Rocha JJ. Jejunal diverticulitis as a cause of acute abdomen. J Coloproctol. 2016;36:50-2. doi: I0.1016/j.jcol.20I5.08.007

II. Mohi RS, Moudgil A, Bhatia SK, Seth K, Kaur T. Complicated jejunal diverticulosis: Small bowel volvulus with obstruction. Iran J Med Sci. 2016;4I:548-5I.

12. Khan HS, Ayyaz M. Jejunal diverticulosis presenting as an acute emergency. J Coll Physicians Surg Pak. 2015;25(Suppl.I):S2O-I. doi: 04.2015/JCPSP.S20S2I

I3. Pedraza NF, Roa A. Diverticulosis del yeyuno: complicaciones y manejo; reporte de caso y revisión de la literatura. Rev Colomb Cir. 20I0;25:48-55. 